\title{
Modélisation de la fissuration en contact roulant
}

\author{
Alexey Koltsov ${ }^{1}$, Daniel Boulanger ${ }^{2}, Z_{\text {Zoubir Ayadi }}{ }^{1}$, Michel Nivoit $^{1}$, \\ Jean-Paul Bettembourg ${ }^{3, a}$ et André Galtier ${ }^{4}$ \\ 1 Laboratoire de Science et Génie des Surfaces, LSGS, UMR CNRS 7570, EEIGM, 6 rue Bastien Lepage, BP 630, \\ 54010 Nancy Cedex, France \\ 2 SOGERAIL, 164 rue Foch, 57700 Hayange, France \\ 3 Laboratoire de Développement et d'Étude des Produits Plats, LEDEPP, 17 avenue des tilleuls, BP 70011, \\ 57191 Florange Cedex, France \\ 4 IRSID, Voie Romaine, BP 30320, 57283 Maizières-Lès-Metz, France
}

Reçu le $1^{\text {er }}$ mars 2002, accepté le 2 juillet 2004

\begin{abstract}
Résumé - Le défaut de fatigue de contact des rails dénommé « head-check » comporte après amorçage une phase de propagation en mode II débouchant soit sur une bifurcation fatale, soit sur un blocage de la fissure. Pour prévoir au cas par cas les conditions qui peuvent conduire à l'une de ces issues, une modélisation de la fissuration en mode II est nécessaire. Dans cette étude, nous montrons que la méthode des dislocations est idéale pour établir les $\Delta K_{\text {II }}$ qui permettent de décrire le comportement de la fissure en utilisant les paramètres de la loi de Paris. Nous analysons ainsi notamment l'effet de proximité dans le cas de paires de fissures, le rôle du frottement entre les faces de celles-ci et sa synergie avec les contraintes résiduelles.

Mots clés : Contact roulant / fissure / méthode des dislocations / modélisation

Abstract - Modelling of crack growth in rolling contact. The so-called "head-check" defect in rolling contact of rails includes a stage of fatigue crack initiation followed by coplanar shear mode crack growth. The end of this stage is bifurcation and failure or crack arrest. We use the distribution of dislocations method to evaluate the values of $\Delta K_{\text {II }}$ which enters the Paris law and allows to predict the behaviour of the crack. The effect of pairs of cracks, rubbing factor between the faces of the crack, and residual stresses is analysed.
\end{abstract}

Key words: Rolling contact / crack / dislocations method / modelling

\section{Introduction}

Les sociétés de réseaux ferroviaires sont confrontées à des endommagements divers affectant la table des rails qui sont apparus depuis une dizaine d'années et qui sont liés aux sollicitations spécifiques et nouvelles imposées par les conditions d'exploitation. Dans certains cas, on a affaire au défaut dénommé « head-check » concernant principalement les rails occupant la position haute dans les dévers de courbes. Un exemple est illustré en figure 1.

L'endommagement comporte un stade préalable au cours duquel une série d'amorces apparaît au sein des couches superficielles soumises à l'écrouissage dû aux contraintes normales, et qui est exacerbé par les contraintes tangentielles exercées par les roues. La synthèse des observations menées sur les divers

\footnotetext{
a Auteur correspondant :

Zoubir.Ayadi@eeigm.inpl-nancy.fr
}

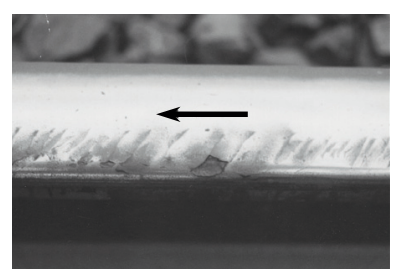

vue de dessus
Sens de roulement

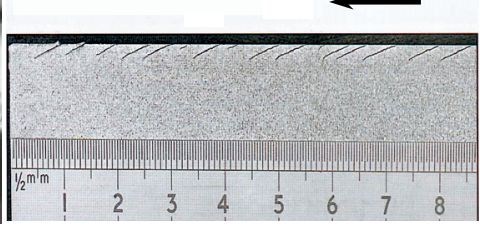

vue en coupe
Fig. 1. Amorçage et propagation du défaut dit de « headchecks $\gg$.

« head-checks » rencontrés conduit au constat que l'angle des fissures et leur espacement sont spécifiques aux tronçons particuliers sur lesquels on les rencontre, voir la figure 1 .

Le stade suivant de l'endommagement est celui de la propagation des fissures. Au cours de cette phase, on peut 
Nomemclature

\begin{tabular}{|c|c|}
\hline$a:$ & longueur d'une fissure \\
\hline$b:$ & vecteur de Burgers \\
\hline$b_{1}:$ & composante réelle du vecteur de Burgers \\
\hline$b_{2}:$ & composante imaginaire du vecteur de Burgers \\
\hline$b_{\ell}:$ & composante longitudinale du vecteur de Burgers \\
\hline$b_{\mathrm{t}}:$ & composante transversale du vecteur de Burgers \\
\hline$c:$ & demi-longueur de contact \\
\hline$d:$ & distance entre deux fissures \\
\hline$f:$ & coefficient de frottement \\
\hline$p:$ & chargement normal \\
\hline$p_{0}:$ & maximum du chargement normal \\
\hline$q:$ & chargement tangentiel \\
\hline$q_{0}:$ & maximum du chargement tangentiel \\
\hline$r:$ & coordonnée adimensionnelle \\
\hline$r_{\mathrm{A}}:$ & abscisse de l'origine d'une fissure \\
\hline$r_{\mathrm{B}}:$ & abscisse de l'extrémité d'une fissure \\
\hline$s:$ & coordonnée adimensionnelle \\
\hline$x:$ & abscisse le long de la fissure \\
\hline$z:$ & coordonnée complexe \\
\hline$z_{0}:$ & coordonnée complexe \\
\hline$u_{1}:$ & composante réelle du déplacement \\
\hline$u_{2}:$ & composante imaginaire du déplacement \\
\hline$u_{\ell}:$ & composante longitudinale du déplacement \\
\hline$u_{\mathrm{t}}:$ & composante transversale du déplacement \\
\hline$B:$ & densité linéique de dislocation \\
\hline$B_{1}:$ & composante réelle de la densité linéique de dislocation \\
\hline$B_{2}:$ & composante imaginaire de la densité linéique de dislocation \\
\hline$B_{\ell}:$ & composante longitudinale de la densité linéique de dislocation \\
\hline$B_{\mathrm{t}}:$ & composante transversale de la densité linéique de dislocation \\
\hline$F_{\mathrm{I}}:$ & facteur d'intensité de contrainte adimensionnelle en mode I \\
\hline$F_{\text {II }}:$ & facteur d'intensité de contrainte adimensionnelle en mode II \\
\hline G : & module de Coulomb du matériau \\
\hline$K_{\mathrm{I}}:$ & facteur d'intensité de contrainte en mode I \\
\hline$K_{\mathrm{II}}:$ & facteur d'intensité de contrainte en mode II \\
\hline$\Delta K_{\mathrm{I}}:$ & amplitude du facteur d'intensité de contrainte en mode I \\
\hline$\Delta K_{\mathrm{II}}:$ & amplitude du facteur d'intensité de contrainte en mode II \\
\hline$\Delta K_{\mathrm{IS}}:$ & amplitude seuil du facteur d'intensité de contrainte en mode I \\
\hline$\Delta K_{\mathrm{IIS}}:$ & amplitude seuil du facteur d'intensité de contrainte en mode II \\
\hline$K_{\ell}:$ & noyau \\
\hline$N:$ & nombre de cycles \\
\hline$R:$ & rayon d'une fissure semi-circulaire \\
\hline$U:$ & vecteur déplacement \\
\hline$\varphi_{\ell}:$ & composante longitudinale de la densité linéique de dislocation \\
\hline$\mu_{\mathrm{f}}:$ & coefficient de frottement entre les faces de la fissure \\
\hline$\nu:$ & coefficient de Poisson du matériau \\
\hline$\theta:$ & angle d'inclinaison d'une fissure \\
\hline$\sigma_{\ell \mathrm{t}}:$ & contrainte de cisaillement induite par les dislocations \\
\hline$\sigma_{\ell \mathrm{t}}^{\text {chargement }}$ & contrainte de cisaillement induite par le chargement \\
\hline$\sigma_{\mathrm{tt}}^{\text {chargement }}$ & contrainte normale induite par le chargement \\
\hline$\sigma_{\text {res }}:$ & contrainte résiduelle \\
\hline$\omega:$ & fonction de contrainte \\
\hline$\omega^{\prime}:$ & dérivée première de la fonction de contrainte \\
\hline$\omega^{\prime \prime}:$ & dérivée seconde de la fonction de contrainte \\
\hline$\Omega:$ & fonction de contrainte \\
\hline$\Omega^{\prime}:$ & dérivée première de la fonction de contrainte \\
\hline$\Omega^{\prime \prime}:$ & dérivée seconde de la fonction de contrainte \\
\hline$\zeta:$ & abscisse le long de la fissure \\
\hline$\Im$ : & partie imaginaire \\
\hline$\Re:$ & partie réelle \\
\hline
\end{tabular}




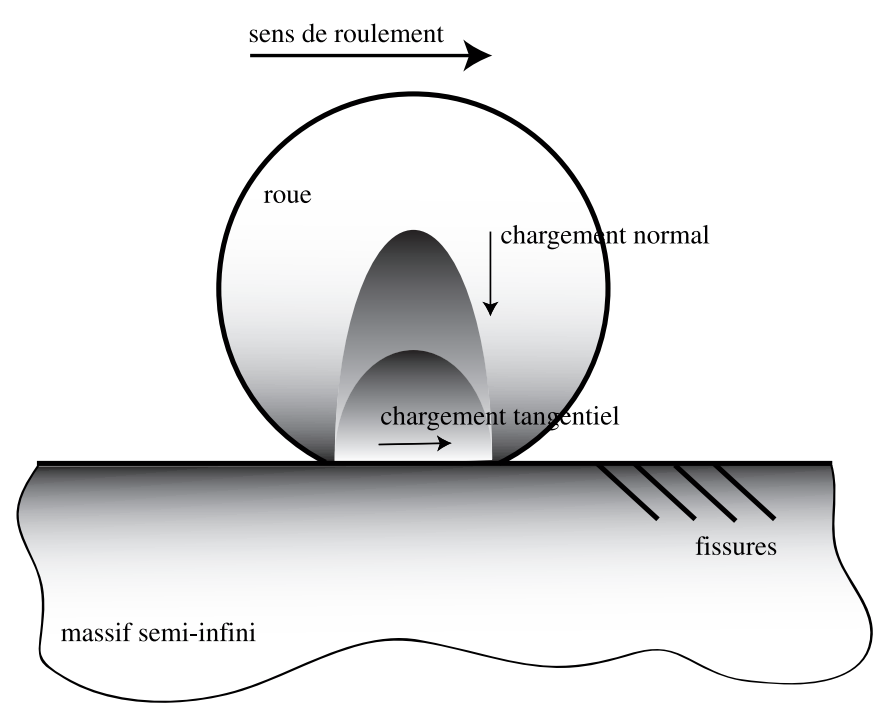

Fig. 2. Configuration du contact en présence d'amorces de fatigue.

voir les fissures adopter des comportements divers, décrits par Miller [1]. Nous retiendrons pour notre part deux illustrations extrêmes de propagation des fissures : soit celles-ci se développent jusqu'à ce que leur longueur devienne suffisante pour qu'apparaisse une bifurcation en mode d'ouverture, entraînant la rupture du rail, soit elles se bloquent au terme d'une certaine progression.

La prédiction du comportement qu'on doit attendre d'une fissure, jusqu'à la fatale ou au blocage éventuel, est utile pour guider la politique de maintenance des voies, elle-même motivée par des préoccupations évidentes de sécurité et d'exploitation.

Le but de la présente étude dans la poursuite de cet objectif est la modélisation de la propagation de fissures dans le prolongement d'amorces, afin de cerner les paramètres majeurs qui la gouvernent, propres au matériau et aux sollicitations imposées. La description géométrique (longueur, inclinaison) de l'amorce simple ou multiple dont le rail est porteur est une donnée du problème, la naissance de cette amorce étant un autre sujet qui a fait l'objet de nombreuses publications, voir Galtier [2]. Nous admettrons que la phase de propagation a lieu en cisaillement de sorte qu'elle est principalement gouvernée par l'amplitude $\Delta K_{\text {II }}$ du facteur d'intensité de contrainte $K_{\text {II }}$ en mode II, hypothèse justifiée par le fait que les pressions de contact importantes inhibent l'ouverture des fissures.

La modélisation mécanique adopte la représentation simplifiée du contact $2 \mathrm{D}$, voir la figure 2, qui permet comme nous le verrons plus loin d'appliquer la méthode des dislocations proposée initialement par Muskhelishvili [3] et grandement enrichie par l'école anglo-américaine dans les années 80. Cette méthode est puissante, car elle permet, au prix d'une mise en œuvre très simple de calculer avec précision les facteurs d'intensité de contrainte aux extrémités d'une fissure et d'en déduire leurs variations au cours d'un cycle. Par ailleurs, elle permet de déterminer facilement les champs de contraintes perturbés par la fissure. Cette méthode constitue une alternative analytico-numérique pertinente aux calculs par éléments finis.

La section 2 est consacrée à quelques rappels sur la méthode des dislocations et présente les principales relations qui conduisent aux répartitions des contraintes et aux facteurs d'intensité de contrainte $K_{\text {II }}$ aux extrémités d'une et de deux fissures. Les résultats sont validés par comparaison à ceux obtenus par éléments finis sur ABAQUS.

La section 3 donne quelques informations sur les évolutions de $\Delta K_{\text {II }}$ en fonction des facteurs géométriques et mécaniques de la configuration du contact entre la roue et le rail. Le rail est représenté comme un massif plan semi-infini contenant une ou plusieurs fissures connues : cette hypothèse est couramment admise vu les faibles dimensions des fissures devant celles du champignon du rail. Ce massif est soumis à un chargement normal $p$ et tangentiel $q$ de forme donnée : on peut lui superposer un champ de contraintes résiduelles connu, et si on le souhaite, introduire des contraintes de flexion. On analyse successivement :

- l'influence de l'inclinaison de la fissure : les résultats sont comparés à ceux obtenus en configuration 3D par Murakami [4],

- l'influence d'une fissure sur une autre,

- l'influence d'un chargement tangentiel,

- l'influence du frottement entre les lèvres de la fissure,

- l'influence des contraintes résiduelles.

Connaissant les caractéristiques intrinsèques de fissuration par cisaillement du matériau données par la loi de Paris en mode II ainsi que le seuil $\Delta K_{\text {IIs }}$, la section 4 montre comment la méthode de calcul proposée peut être mise à profit pour résoudre tout problème spécifique d'endommagement susceptible d'affecter la table d'un rail, et en particulier d'évaluer suffisamment tôt la durée de vie résiduelle ou la chance de blocage de la fissure.

\section{Rappel de la méthode des dislocations}

La méthode des dislocations reprend de la modélisation microstructurale des dislocations le principe du déplacement relatif de deux éléments de matière, initialement voisins, pour l'appliquer à l'étude du comportement d'un matériau contenant une fissure et soumis à un champ de contraintes donné.

\subsection{Représentation complexe d'un problème d'élasticité plane}

Muskhelishvili [3] a montré qu'un problème d'élasticité plane pouvait se résoudre en introduisant deux fonctions de contraintes complexes analytiques $\Omega(z)$ et $\omega(z)$ où $z$ est la coordonnée complexe du point étudié, voir la figure 3 . En notant $U=u_{1}+\mathrm{i} u_{2}$ le déplacement complexe, les équations de Navier sont satisfaites si on pose :

$$
2 G U=A \Omega(z)-z \overline{\Omega^{\prime}(z)}-\overline{\omega(z)}
$$




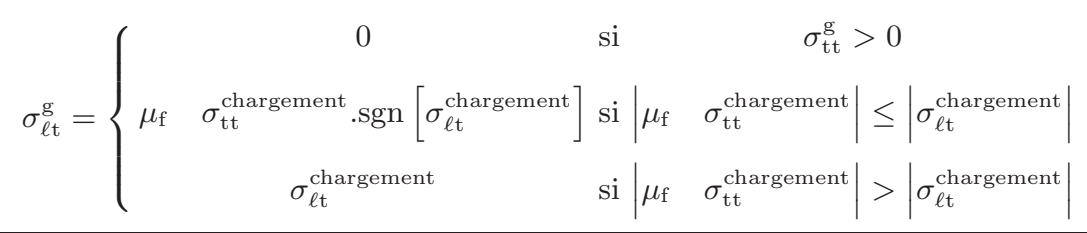

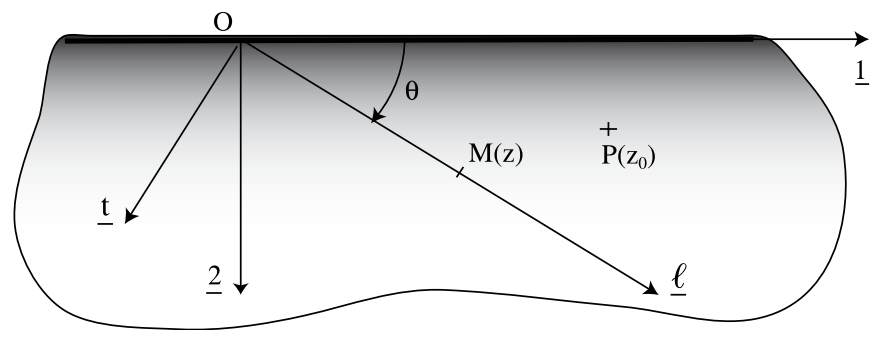

Fig. 3. Notations utilisées pour la description du massif semiinfini.

où $A=3-4 \nu$ pour un problème de déformation plane et $A=\frac{3-\nu}{1+\nu}$ pour un problème de contraintes planes sachant que $\nu$ et $G$ sont respectivement le coefficient de Poisson et le module de Coulomb du matériau.

En coordonnées cylindriques, le déplacement est noté $U=u_{\ell}+\mathrm{i} u_{\mathrm{t}}$ et les contraintes s'expriment par les relations suivantes :

$$
\begin{aligned}
2 G U & =\left[A \Omega(z)-z \overline{\Omega^{\prime}(z)}-\overline{\omega(z)}\right] \mathrm{e}^{-\mathrm{i} \theta} \\
\sigma_{\ell \ell} & =\Re\left[\left(\Omega^{\prime}(z)+\overline{\Omega^{\prime}(z)}\right)-\left(\bar{z} \Omega^{\prime \prime}(z)-\omega^{\prime}(z)\right) \mathrm{e}^{2 \mathrm{i} \theta}\right] \\
\sigma_{\mathrm{tt}} & =\Re\left[\left(\Omega^{\prime}(z)+\overline{\Omega^{\prime}(z)}\right)+\left(\bar{z} \Omega^{\prime \prime}(z)-\omega^{\prime}(z)\right) \mathrm{e}^{2 \mathrm{i} \theta}\right] \\
\sigma_{\ell \mathrm{t}} & =\Im\left[\left(\bar{z} \Omega^{\prime \prime}(z)-\omega^{\prime}(z)\right) \mathrm{e}^{2 \mathrm{i} \theta}\right]
\end{aligned}
$$

\subsection{Cas d'une fissure}

Selon Bower [5], les contraintes au point $M(z)$ induites par une dislocation de vecteur de Burgers $b=b_{1}+\mathrm{i} b_{2}$ en $P\left(z_{0}\right)$ se déduisent des relations (2)-(5) en précisant la forme des fonctions de contraintes $\Omega(z)$ et $\omega(z)$. Dans le cas d'un massif infini elles s'écrivent :

$$
\begin{aligned}
& \Omega(z)=\Omega_{0}(z)=\alpha \ln \left(z-z_{0}\right) \\
& \omega(z)=\omega_{0}(z)=\bar{\alpha} \ln \left(z-z_{0}\right)-\frac{\alpha}{z-z_{0}} \overline{z_{0}}
\end{aligned}
$$

avec :

$$
\alpha=\frac{G}{4 \pi(1-\nu)}\left(-b_{2}+\mathrm{i} b_{1}\right)=\frac{G}{4 \pi(1-\nu)}\left(-b_{\mathrm{t}}+\mathrm{i} b_{\ell}\right) \mathrm{e}^{\mathrm{i} \theta}
$$

Dans le cas d'un massif semi-infini, voir la figure 4, elles s'écrivent :

$$
\begin{aligned}
& \Omega(z)=\Omega_{0}(z)-z \overline{\Omega_{0}^{\prime}(\bar{z})}-\overline{\omega_{0}(\bar{z})} \\
& \omega(z)=\omega_{0}(z)+z \overline{\omega_{0}^{\prime}(\bar{z})}-\overline{\Omega_{0}(\bar{z})}+z \overline{\Omega_{0}^{\prime}(\bar{z})}+z^{2} \overline{\Omega_{0}^{\prime \prime}(\bar{z})}
\end{aligned}
$$

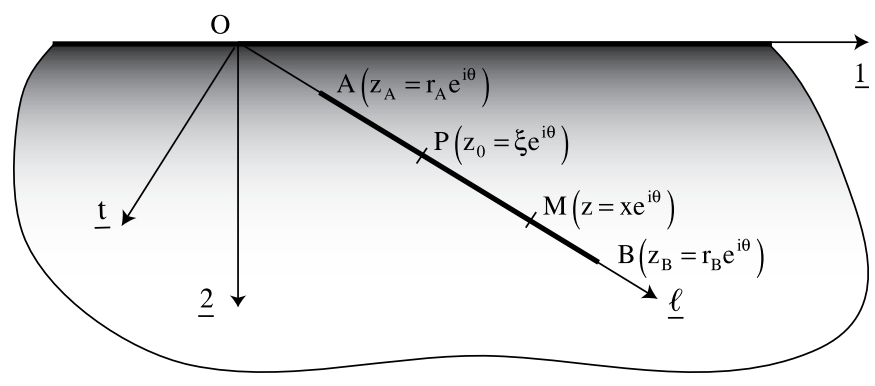

Fig. 4. Notations utilisées pour la description d'une fissure.

dans lesquelles $\Omega_{0}(z)$ et $\omega_{0}(z)$ ont les expressions données par $(6),(7)$ et $(8)$.

Les travaux effectués sur la modélisation des fissures par Muskhelishvili [3] et poursuivis par Erdogan [6] ont été maintes fois exploités dans les années 80. L'article de Hills [7] en constitue une bonne synthèse accompagnée d'une bibliographie complète.

Selon ces travaux, la fissure est assimilée à une distribution linéique de dislocations de densité $B\left(z_{0}\right)=B_{\ell}\left(z_{0}\right)+\mathrm{i} B_{\mathrm{t}}\left(z_{0}\right)$ en $P\left(z_{0}\right)$, voir la figure 4 . On suppose par la suite que $B_{\mathrm{t}}\left(z_{0}\right)=0$, la fissuration se faisant, selon nos hypothèses, par cisaillement. La densité $B_{\ell}\left(z_{0}\right)$ doit satisfaire deux conditions :

- la première exprime que globalement le vecteur de Burgers est nul pour une fissure non débouchante :

$$
\int_{z_{\mathrm{A}}}^{z_{\mathrm{B}}} B_{\ell}\left(z_{0}\right) \mathrm{d} z_{0}=0
$$

Par contre, pour une fissure débouchante en A on doit écrire que les facteurs d'intensité de contraintes sont nuls en ce point, en particulier :

$$
K_{\mathrm{II}}\left(z_{\mathrm{A}}\right)=0
$$

- la seconde exprime les conditions aux limites le long de la fissure et porte sur la contrainte de cisaillement global en $M(z)$ :

$$
\sigma_{\ell \mathrm{t}}^{\mathrm{g}}=\sigma_{\ell \mathrm{t}}+\sigma_{\ell \mathrm{t}}^{\mathrm{chargement}}
$$

où $\sigma_{\ell t}$ est la contrainte induite par les dislocations, et $\sigma_{\ell \mathrm{t}}^{\text {chargement }}$ la contrainte imposée par le chargement. Trois cas doivent être envisagés selon que l'on considère que les faces de la fissure sont libres ou non :

$$
\text { voir l'équation (14) ci-dessus }
$$

où $\mu_{\mathrm{f}}$ est le coefficient de frottement entre les faces de la fissure selon la loi de Coulomb. 
Le problème est alors le calcul de la densité de dislocations $B_{\ell}\left(z_{0}\right)$ qui doit satisfaire en tout point $M(z)$ de la fissure :

$$
\sigma_{\ell \mathrm{t}}=\sigma_{\ell \mathrm{t}}^{\mathrm{g}}-\sigma_{\ell \mathrm{t}}^{\text {chargement }}
$$

avec :

$$
\sigma_{\ell \mathrm{t}}=\Re\left[\int_{z_{\mathrm{A}}}^{z_{\mathrm{B}}} \frac{G}{4 \pi(1-\nu)} K_{\ell}\left(z, z_{0}\right) B_{\ell}\left(z_{0}\right) \mathrm{d} z_{0}\right]
$$

où $K_{\ell}\left(z, z_{0}\right)$ est une fonction a priori connue qui dépend de la géométrie et que l'on nomme «noyau». Dans le cas d'un massif semi-infini :

$$
K_{\ell}\left(z, z_{0}\right)=C \mathrm{e}^{3 \mathrm{i} \theta}+D \mathrm{e}^{\mathrm{i} \theta}
$$

avec :

$$
C=\frac{1}{z-\overline{z_{0}}}-\frac{\bar{z}-\overline{z_{0}}}{\left(z-z_{0}\right)}-\frac{z-\bar{z}}{\left(z-\overline{z_{0}}\right)^{2}}
$$

et :

$$
D=-\frac{1}{z-z_{0}}-\frac{z_{0}+2 \bar{z}-3 z}{\left(z-z_{0}\right)}-\frac{2(z-\bar{z})\left(z-z_{0}\right)}{\left(z-\overline{z_{0}}\right)^{2}}
$$

Sachant que :

$$
z=x \mathrm{e}^{\mathrm{i} \theta} \quad \text { et } \quad z_{0}=\xi \mathrm{e}^{\mathrm{i} \theta}
$$

en introduisant les coordonnées adimensionnelles $s$ et $r$ telles que :

$$
\begin{aligned}
& x=\frac{r_{\mathrm{B}}-r_{\mathrm{A}}}{2} s+\frac{r_{\mathrm{B}}+r_{\mathrm{A}}}{2} \\
& \xi=\frac{r_{\mathrm{B}}-r_{\mathrm{A}}}{2} r+\frac{r_{\mathrm{B}}+r_{\mathrm{A}}}{2}
\end{aligned}
$$

en posant :

$$
K_{\ell}(x, \xi)=\frac{r_{\mathrm{B}}-r_{\mathrm{A}}}{2} K_{\ell}(s, r)
$$

et en effectuant le changement de fonction suivant pour tenir compte de ce que la fissure est une singularité d'ordre $1 / 2$ :

$$
B_{\ell}(r)=\frac{4 \pi(1-\nu)}{G} \frac{\varphi_{\ell}(r)}{\sqrt{1-r^{2}}}
$$

la relation (14) s'écrit :

$$
\sigma_{\ell \mathrm{t}}=\int_{-1}^{1} K_{\ell}(s, r) \frac{\varphi_{\ell}(r)}{\sqrt{1-r^{2}}} \mathrm{~d} r
$$

De la même façon, les relations (9) et (10) deviennent respectivement :

$$
\begin{array}{r}
\int_{-1}^{1} \frac{\varphi_{\ell}(r)}{\sqrt{1-r^{2}}} \mathrm{~d} r=0 \\
\varphi_{\ell}(-1)=0
\end{array}
$$

sachant que les facteurs d'intensité de contraintes en pointes de fissure non débouchante s'écrivent :

$$
\begin{aligned}
& K_{\mathrm{II}}(\mathrm{a})=\pi \varphi_{\ell}(-1) \sqrt{2 \pi\left(r_{\mathrm{B}}-r_{\mathrm{A}}\right)} \\
& K_{\mathrm{II}}(\mathrm{b})=\pi \varphi_{\ell}(1) \sqrt{2 \pi\left(r_{\mathrm{B}}-r_{\mathrm{A}}\right)}
\end{aligned}
$$

Ainsi, la fonction $\varphi_{\ell}(r)$ peut être déterminée après avoir discrétisé les relations (25), (26) ou (27) et en tenant compte de (15). On applique la méthode reprise par Erdogan [5] qui pose :

$$
\begin{aligned}
& s_{k}=\cos \left(\frac{\mathrm{k} \pi}{n}\right) k=1, \ldots, n-1 \\
& r_{i}=\cos \left(\frac{(2 i-1) \pi}{2 n}\right) i=1, \ldots, n
\end{aligned}
$$

L'équation (15) s'écrit :

$$
\begin{aligned}
& \sum_{i=1}^{n} K_{\ell}\left(s_{k}, r_{i}\right) \varphi_{\ell}\left(r_{i}\right)= \\
& \frac{1}{\sin \frac{\pi}{n}}\left(\sigma_{\ell \mathrm{t}}^{\mathrm{g}}-\sigma_{\ell \mathrm{t}}^{\text {chargement }}\right) k=1, \ldots, n-1
\end{aligned}
$$

De même, l'équation (26) devient :

$$
\sum_{i=1}^{n} \varphi_{\ell}\left(r_{i}\right)=0
$$

L'équation (27) est inchangée :

$$
\varphi_{\ell}\left(r_{n}\right)=0
$$

Les équations (32) et (34) ou (32) et (33) selon que la fissure est débouchante ou non forment un système linéaire de $n$ équations à $n$ inconnues dont la résolution donne $\varphi_{\ell}\left(r_{i}\right)$ pour $i=1, \ldots, n$. Les facteurs d'intensité de contraintes se déduisent par extrapolation selon les formules de Krenk [8] :

$$
\begin{aligned}
\varphi_{\ell}(-1) & =\frac{1}{n} \sum_{i=1}^{n} F_{i} \varphi_{\ell}\left(r_{n+1-i}\right) \\
\varphi_{\ell}(1) & =\frac{1}{n} \sum_{i=1}^{n} F_{i} \varphi_{\ell}\left(r_{i}\right) \\
F_{i} & =\frac{\sin \left(\frac{2 n-1}{4 n}(2 i-1) \pi\right)}{\sin \left(\frac{2 i-1}{4 n} \pi\right)}
\end{aligned}
$$

Cette méthode s'applique sans difficulté à une fissure unique, débouchante ou non. Nous examinons maintenant comment on peut l'appliquer à deux fissures.

\subsection{Cas de deux fissures}

Le schéma est donné par la figure 5 . En tout point $M_{1}$ de la première fissure, la relation (15) s'écrit :

$$
\sigma_{\ell \mathrm{t}}^{1}=\sigma_{\ell \mathrm{t}}^{1 \mathrm{~g}}-\sigma_{\ell \mathrm{t}}^{1 \text { chargement }}
$$




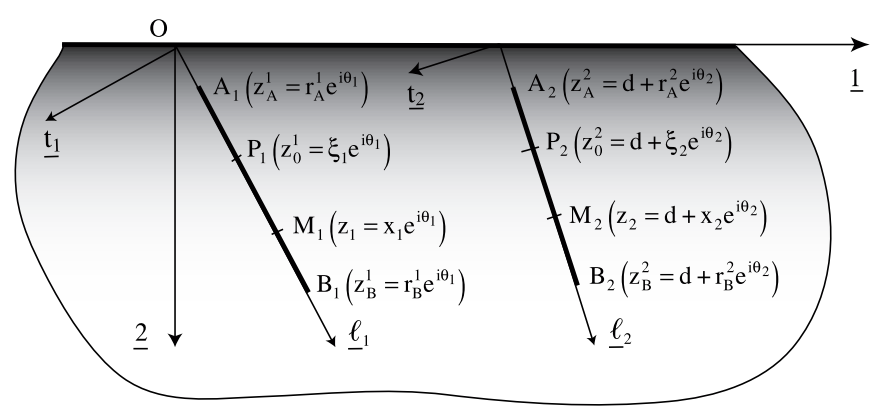

Fig. 5. Notations utilisées pour la description de deux fissures.

avec

$$
\begin{aligned}
\sigma_{\ell \mathrm{t}}^{1}=\int_{r_{\mathrm{A}}^{1}}^{r_{\mathrm{B}}^{1}} & \frac{G}{4 \pi(1-\nu)} K_{\ell}\left(x_{1}, \xi_{1}\right) B_{\ell}^{1}\left(\xi_{1}\right) \mathrm{d} \xi_{1} \\
& +\int_{r_{\mathrm{A}}^{2}}^{r_{\mathrm{B}}^{2}} \frac{G}{4 \pi(1-\nu)} K_{\ell}\left(x_{1}, \xi_{2}\right) B_{\ell}^{2}\left(\xi_{2}\right) \mathrm{d} \xi_{2}
\end{aligned}
$$

où le second terme représente l'influence de la deuxième fissure. De même, en tout point de la seconde fissure, on peut écrire :

$$
\sigma_{\ell \mathrm{t}}^{2}=\sigma_{\ell \mathrm{t}}^{2 \mathrm{~g}}-\sigma_{\ell \mathrm{t}}^{2 \text { chargement }}
$$

avec

$$
\begin{aligned}
\sigma_{\ell \mathrm{t}}^{2}=\int_{r_{\mathrm{A}}^{2}}^{r_{\mathrm{B}}^{2}} \frac{G}{4 \pi(1-\nu)} K_{\ell}\left(x_{2}, \xi_{2}\right) B_{\ell}^{2}\left(\xi_{2}\right) d \xi_{2} \\
\quad+\int_{r_{\mathrm{A}}^{1}}^{r_{\mathrm{B}}^{1}} \frac{G}{4 \pi(1-\nu)} K_{\ell}\left(x_{2}, \xi_{1}\right) B_{\ell}^{1}\left(\xi_{1}\right) \mathrm{d} \xi_{1}
\end{aligned}
$$

où le second terme représente l'influence de la première fissure.

Si on adopte les mêmes discrétisations sur les deux fissures, on obtient finalement :

$$
\begin{aligned}
\sum_{i=1}^{n}\left[K_{\ell}\left(s_{k}^{1}, r_{i}^{1}\right) \varphi_{\ell}^{1}\left(r_{i}^{1}\right)+\right. & \left.K_{\ell}\left(s_{k}^{1}, r_{i}^{2}\right) \varphi_{\ell}^{2}\left(r_{i}^{2}\right)\right]= \\
& \frac{1}{\sin \frac{\pi}{n}}\left(\sigma_{\ell t}^{1 \mathrm{~g}}-\sigma_{\ell \mathrm{t}}^{1 \text { chargement }}\right)
\end{aligned}
$$

où $i=1, \ldots, n$ et $k=1, \ldots, n-1$, avec :

$$
\varphi_{\ell}^{1}\left(r_{n}^{1}\right)=0 \quad \text { ou } \quad \sum_{i=1}^{n} \varphi_{\ell}^{1}\left(r_{i}^{1}\right)=0
$$

selon que la première fissure débouche ou pas

$$
\begin{aligned}
\sum_{i=1}^{n}\left[K_{\ell}\left(s_{k}^{2}, r_{i}^{2}\right) \varphi_{\ell}^{2}\left(r_{i}^{2}\right)+\right. & \left.K_{\ell}\left(s_{k}^{2}, r_{i}^{1}\right) \varphi_{\ell}^{1}\left(r_{i}^{1}\right)\right]= \\
& \frac{1}{\sin \frac{\pi}{n}}\left(\sigma_{\ell \mathrm{t}}^{2 \mathrm{~g}}-\sigma_{\ell \mathrm{t}}^{2 \text { chargement }}\right)
\end{aligned}
$$

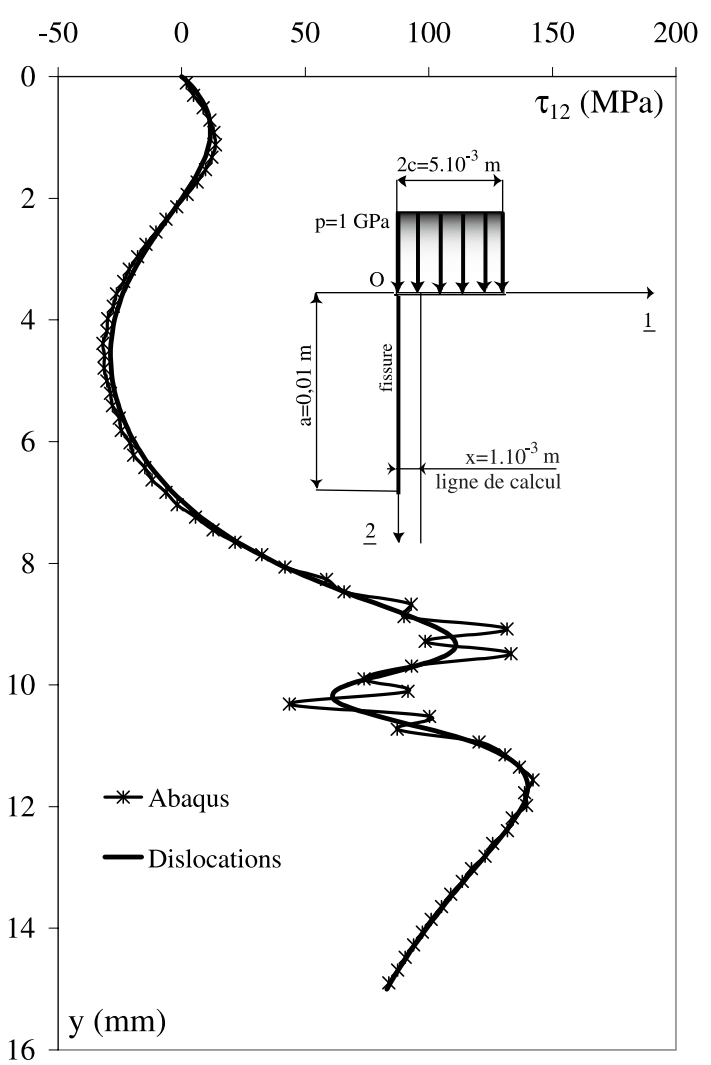

Fig. 6. Comparaison entre les contraintes de cisaillement $\tau_{12}$ calculées par la méthode des dislocations et par éléments finis sur ABAQUS.

où $i=1, \ldots, n$ et $k=1, \ldots, n-1$, et

$$
\varphi_{\ell}^{2}\left(r_{n}^{2}\right)=0 \quad \text { ou } \quad \sum_{i=1}^{n} \varphi_{\ell}^{2}\left(r_{i}^{2}\right)=0
$$

selon que la deuxième fissure débouche ou pas.

Les équations (42)-(45) forment alors un système de $2 n$ équations à $2 n$ inconnues dont la résolution donne $\varphi_{\ell}^{1}\left(r_{i}\right)$ et $\varphi_{\ell}^{2}\left(r_{i}\right)$ pour $i=1, \ldots, n$. Les facteurs d'intensité de contraintes se déduisent par extrapolation selon les formules de Krenk [8].

Nous donnerons à la section 3 des exemples d'application de cette méthode appliquée à des situations réelles de contact.

\subsection{Champs de contraintes perturbés par une fissure}

La méthode présente conduit à la connaissance de la distribution des dislocations le long de la fissure. Le champ de contrainte perturbé par la fissure est la superposition des champs produits par le chargement extérieur et par l'ensemble des dislocations.

On illustre sur la figure 6 le résultat obtenu dans le cas d'un massif semi-infini présentant une fissure verticale débouchante et soumis à un chargement uniforme de densité surfacique $1 \mathrm{GPa}$ réparti sur une longueur 
de $5 \times 10^{-3} \mathrm{~m}$. L'application de la méthode des dislocations montre la précision qu'on peut attendre de cette méthode comparativement à un calcul par éléments finis sur ABAQUS.

\section{Influence sur $K_{\| I}$ des paramètres du contact}

La formulation dans le plan complexe qui vient d'être présentée conduit, entre autre, à la détermination des facteurs d'intensité de contraintes : elle permet donc d'étudier les rôles respectifs des nombreux paramètres intervenant dans la fissuration.

\subsection{Influence de l'inclinaison de la fissure}

L'effet de l'inclinaison est étudié en comparant les évolutions de $K_{\text {II }}$ au cours d'un cycle entre une fissure débouchante longue de $a=2,5 \mathrm{~mm}$ inclinée à $45^{\circ}$ et une fissure verticale de même type et de même longueur. Le chargement est hertzien avec $p_{0}=1,5 \mathrm{GPa}$ et $q_{0}=0,1 p_{0}$, réparti sur une longueur de contact $2 c=5 \mathrm{~mm}$. Les lèvres de la fissure sont supposées libres de frottement.

Les résultats sont présentés à la figure 7 . On voit que l'inclinaison de la fissure joue sur la forme des courbes ainsi que sur la variation $\Delta K_{\text {II }}$ entre les valeurs extrêmes, l'inclinaison à $45^{\circ}$ donnant un $\Delta K_{\mathrm{II}} 32 \%$ plus élevé qu'en verticale.

Les conditions de chargement retenues dans ce calcul ont été choisies afin d'autoriser la comparaison entre nos résultats concernant une fissure $2 \mathrm{D}$ et ceux figurant dans le manuel de Murakami [4] portant sur une fissure semicirculaire de rayon $R=a$, représentée sur la figure, traitée en 3D par éléments finis.

La comparaison entre les coefficients $F_{\text {II }}=$ $K_{\mathrm{II}} / p_{0} \sqrt{\pi R}$ reportés sur les figures 9 et 10 , sur fissure verticale d'une part, à $45^{\circ}$ d'autre part, montre qu'il y a lieu de tenir compte d'un facteur d'environ 0,7 pour passer d'une fissure 2D à une fissure semi-circulaire 3D. Le calcul 2D, seul autorisé actuellement par la méthode des dislocations, est donc une très bonne approche de la réalité $3 \mathrm{D}$ si l'on accepte d'appliquer un facteur tiré de la comparaison entre les calculs effectués en 3D dans des situations précises, Murakami [4], et ceux obtenus par la présente méthode dans les mêmes conditions.

Les courbes précédentes représentant l'évolution de $K_{\text {II }}$ en fonction de la position de la roue sur un cycle complet mettent en évidence la rapide variation de $K_{\text {II }}$ d'une valeur extrême à l'autre lorsque la roue franchit la fissure. L'écart maximal $\Delta K_{\text {II }}$ qui en résulte est la caractéristique utile pour évaluer l'avancée de la fissure à chaque cycle : c'est ce paramètre que nous étudions dans ce qui suit.

\subsection{Influence d'une double fissure}

L'effet de double fissure que nous étudions maintenant porte sur une paire de fissures débouchantes verticales

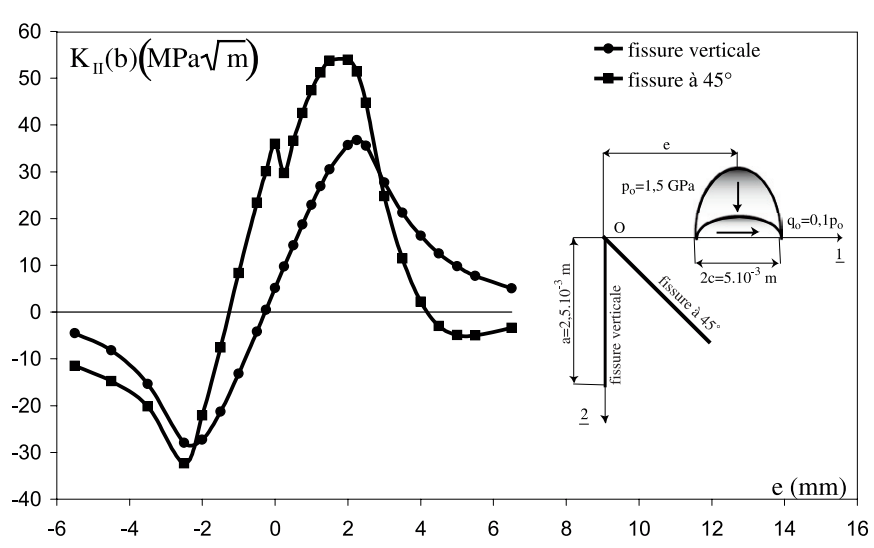

Fig. 7. Effet de l'inclinaison de la fissure sur l'évolution du facteur d'intensité de contrainte $K_{\text {II }}$ au cours d'un cycle.

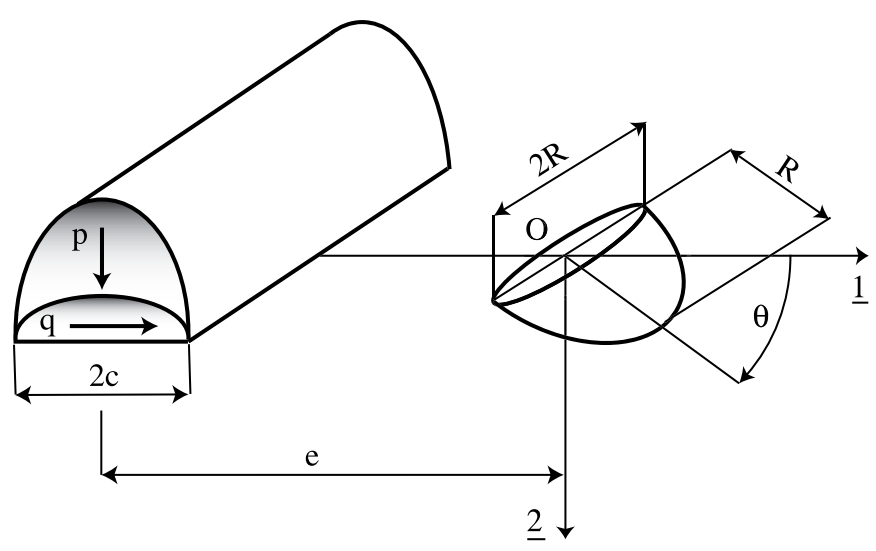

Fig. 8. Schéma de la fissure semi-circulaire étudiée par Murakami [4].

dont nous faisons varier l'écartement $d$. Le chargement retenu est celui évoqué à la section 2.4 , chargement uniforme de densité surfacique $p=1 \mathrm{GPa}$ réparti sur une longueur de $2 c=5 \times 10^{-3} \mathrm{~m}$.

Les résultats portés en figure 11 montrent l'évolution de l'amplitude $\Delta K_{\text {II }}$ en fonction de la longueur a des fissures. L'effet de paire exacerbe les valeurs de $\Delta K_{\text {II }}$ d'autant plus que l'écartement $d$ est faible, allant même, pour $d=1 \mathrm{~mm}$, jusqu'à tripler la valeur maximale atteinte dans le cas d'une fissure unique.

\subsection{Influence d'un chargement tangentiel}

L'effet de l'effort tangentiel est analysé dans le cas d'un chargement hertzien avec $p_{0}=1,5 \mathrm{GPa}$ et $q_{0}=$ $0,3 p_{0}$, réparti sur une longueur de contact $2 c=5 \mathrm{~mm}$. Les résultats, évolution de $\Delta K_{\mathrm{II}}$ en fonction de la longueur $a$ des fissures, sont reportés à la figure 12 pour un coefficient de frottement $f=0,3$.

Dans le cas d'une fissure unique on constate que cet effet ne joue pas sur $\Delta K_{\mathrm{II}}$. Toutefois, l'examen des évolutions de $K_{\text {II }}$ pour chacune des fissures, résultats non reportés dans ce texte, montre qu'elles sont différentes. 


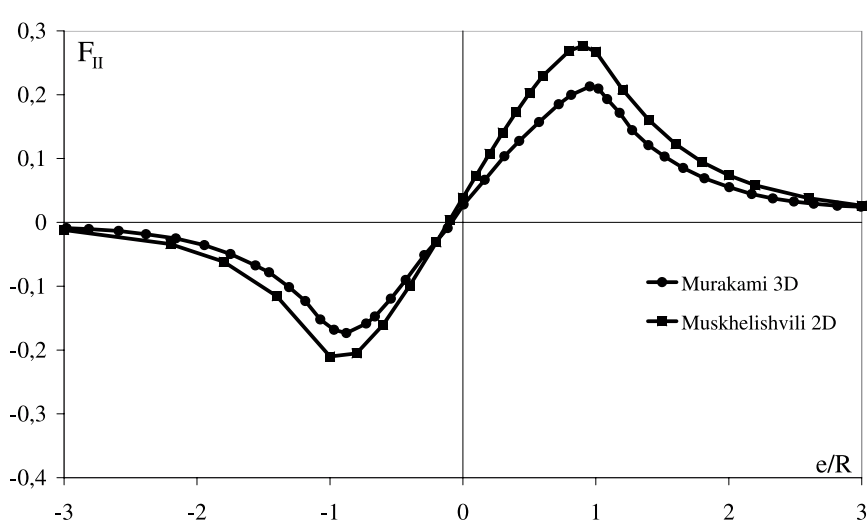

Fig. 9. Comparaison entre les évolutions de $F_{\mathrm{II}}=K_{\mathrm{II}} / p_{0} \sqrt{\pi R}$ obtenues sur fissure 2D (dislocations) et fissure semi-circulaire 3D (Murakami) pour la fissure verticale.

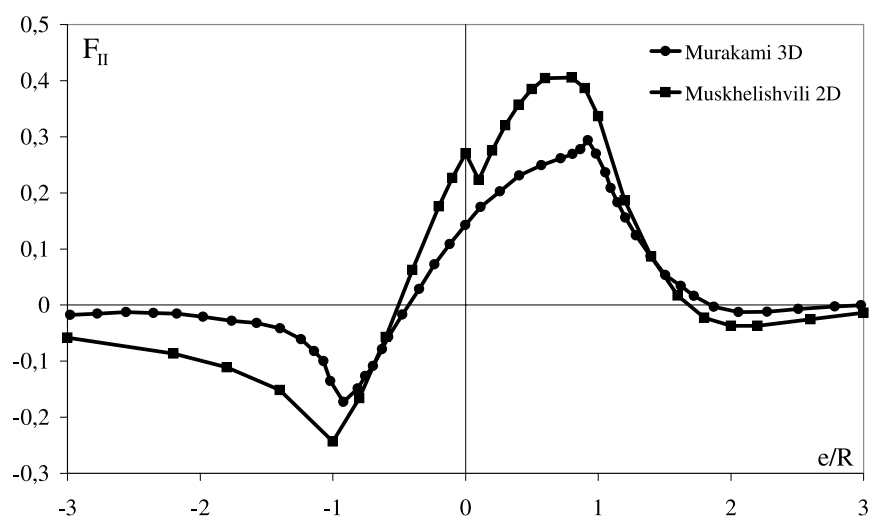

Fig. 10. Comparaison entre les évolutions de $F_{\mathrm{II}}=K_{\mathrm{II}} / p_{0} \sqrt{\pi R}$ obtenues sur fissure 2D (dislocations) et fissure semi-circulaire 3D (Murakami) pour la fissure inclinée.

Dans le cas de paires de fissures séparées de $1 \mathrm{~mm}$ et de $2 \mathrm{~mm}$, on note que le frottement entraîne une dissymétrie qui peut se traduire très rapidement par une différentiation entre les vitesses de propagation de l'une ou l'autre fissure.

\subsection{Influence du frottement entre les lèvres de fissure}

Jusqu'à présent, l'examen des courbes représentant la variation de $\Delta K_{\mathrm{II}}$ en fonction de la longueur $a$ de fissure montre une croissance rapide de $\Delta K_{\text {II }}$ dans les premiers stades de la fissuration, à des niveaux tels que l'apparition d'amorces serait fatale à bref délai, ce qui n'est heureusement pas le cas d'une façon générale. La propagation est donc inhibée par un mécanisme protecteur dont nous n'avons pas encore tenu compte et qui est abondamment décrit dans la littérature, Murakami [4], Hills [6] et O'Regan [9] : le frottement entre les faces de la fissure. Ce frottement est lié à la rugosité des faces due au fait que la fissuration progresse en contournant les barrières

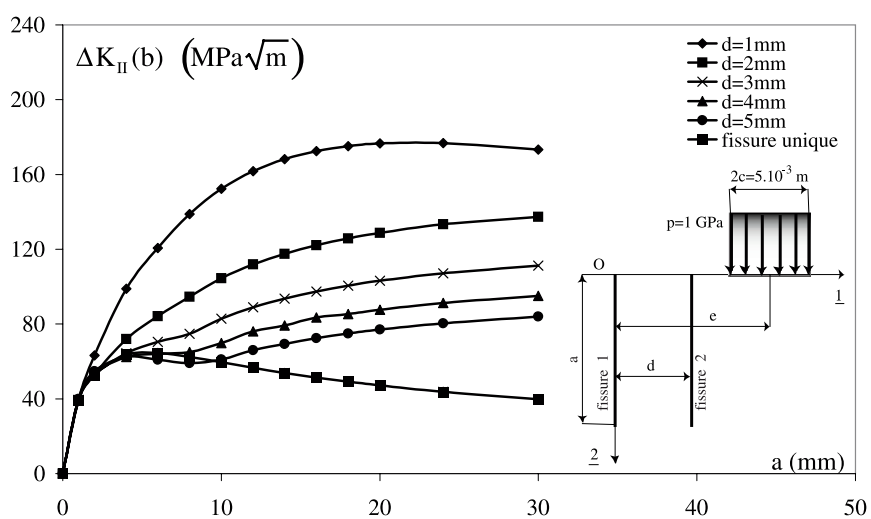

Fig. 11. Effet de l'écartement entre deux fissures sur les évolutions de l'amplitude $\Delta K_{\mathrm{II}}$ du facteur d'intensité de contrainte en fonction de leur longueur.

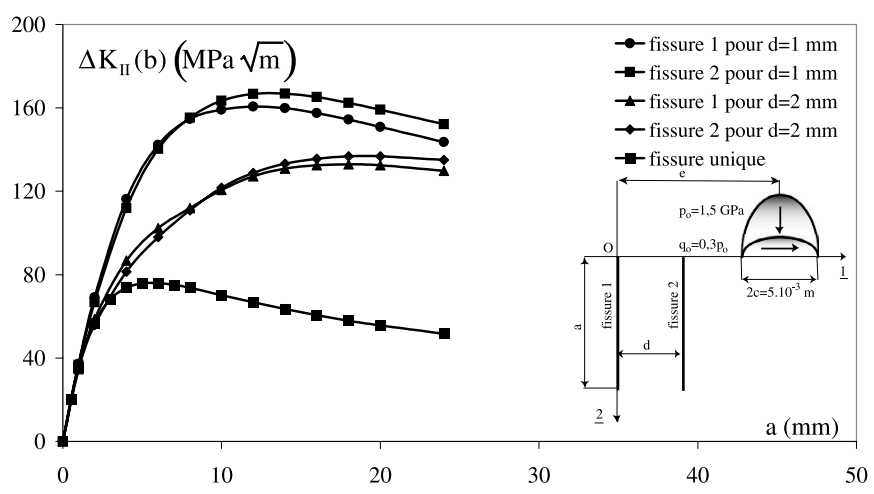

Fig. 12. Effet des sollicitations tangentielles sur les évolutions de l'amplitude $\Delta K_{\text {II }}$ du facteur d'intensité de contrainte en fonction de la longueur des fissures.

microstructurales. Conformément à la loi de Coulomb, ce frottement est défini par le coefficient $\mu_{\mathrm{f}}$, caractéristique de la structure granulaire du matériau.

L'influence du coefficient $\mu_{\mathrm{f}}$ est montrée à la figure 13 qui représente les variations de $\Delta K_{\text {II }}$ en fonction de la longueur $a$ de la fissure pour $\mu_{\mathrm{f}}$ variant de 0 à 0,6 . Le chargement est hertzien, normal pur avec $p_{0}=1,5 \mathrm{GPa}$ et $2 c=5 \times 10^{-3} \mathrm{~m}$. On vérifie que le frottement fait baisser fortement $\Delta K_{\mathrm{II}}$.

\subsection{Influence des contraintes résiduelles}

Nous constatons l'importance du rôle joué par $\mu_{\mathrm{f}}$ et nous pouvons alors imaginer que les contraintes normales qui interviennent en premier lieu dans la résistance au glissement des faces soient elles-mêmes très influencées par la présence ou non de contraintes résiduelles $\sigma_{\text {res }}$ qui s'ajoutent à celles dues au contact.

Nous avons introduit de telles contraintes de compression, au niveau de $\sigma_{\text {res }}=-300 \mathrm{MPa}$. Pour $\mu_{\mathrm{f}}=0,3$, la comparaison est portée à la figure 14 dans le cas d'un chargement hertzien avec $p_{0}=1,5 \mathrm{GPa}$ et $q_{0}=0,3 p_{0}$, 


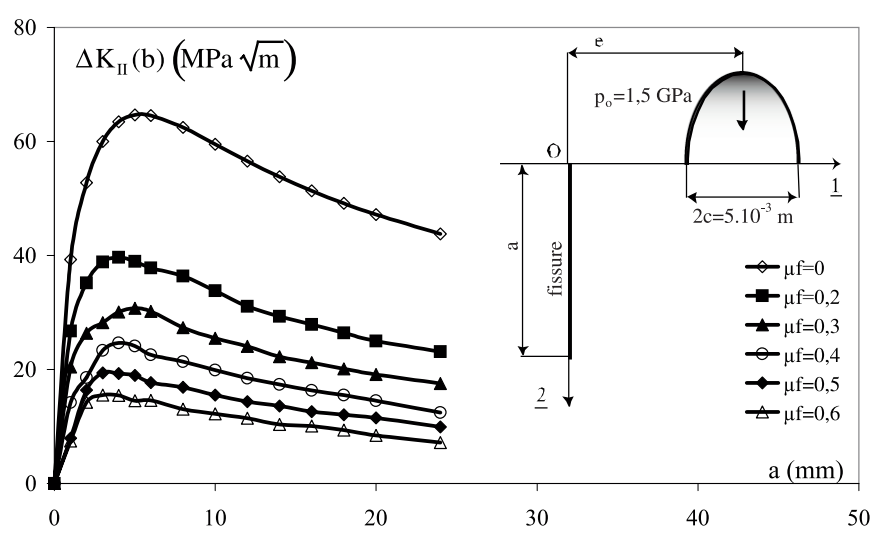

Fig. 13. Effet du coefficient de frottement entre les faces de la fissure sur les évolutions de l'amplitude $\Delta K_{\text {II }}$ du facteur d'intensité de contrainte en fonction de la longueur de la fissure.

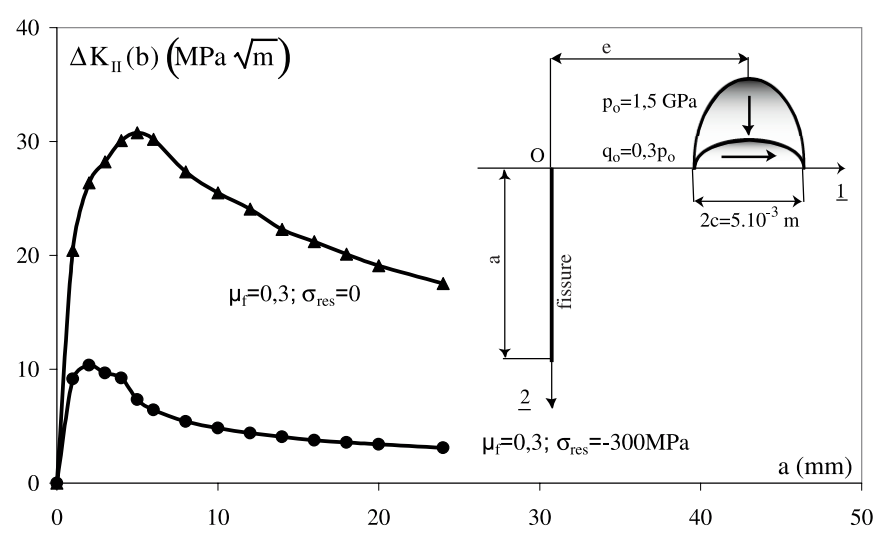

Fig. 14. Effet des contraintes résiduelles sur les évolutions de l'amplitude $\Delta K_{\mathrm{II}}$ du facteur d'intensité de contrainte en fonction de la longueur de la fissure.

réparti sur une longueur de contact $2 c=5 \mathrm{~mm}$. Les résultats, évolution de $\Delta K_{\mathrm{II}}$ en fonction de la longueur $a$ des fissures, montrent que les contraintes résiduelles de compression ont un effet protecteur très important visà-vis de la fissuration puisque les $\Delta K_{\mathrm{II}}$ sont divisés par trois ainsi que la taille de fissure pour laquelle $\Delta K_{\text {II }}$ est maximal.

\section{Discussion}

Les études d'évolution de $\Delta K_{\mathrm{II}}$ abordées précédemment ne visent pas l'objectif de constituer une étude paramétrique complète de la propagation des fissures, mais plutôt de montrer comment l'outil de calcul utilisé, la méthode des dislocations, peut être mis à profit pour résoudre au cas par cas tout problème spécifique d'endommagement susceptible d'affecter la table d'un rail, et en particulier d'évaluer suffisamment tôt la durée de vie résiduelle ou la chance de blocage de la fissure.

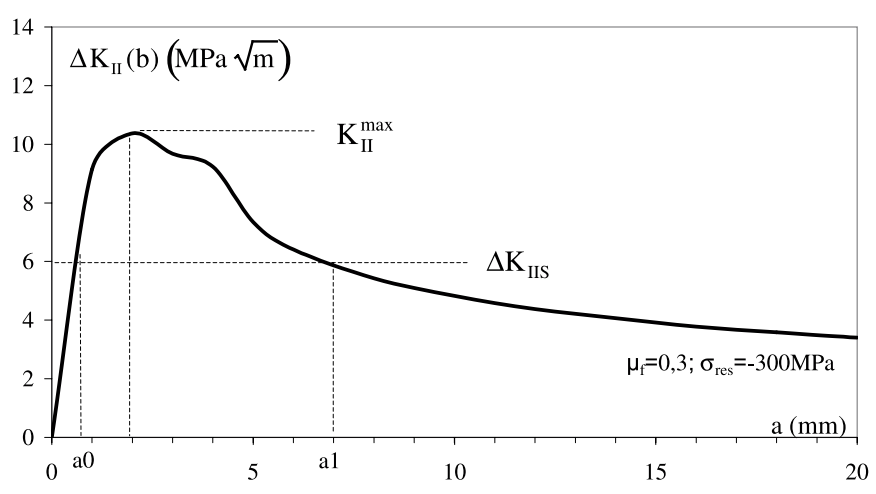

Fig. 15. Description du comportement de la fissure au fur et à mesure de la propagation.

Nous étudions dans cette section, en vue de cet objectif de prédiction, l'exploitation des informations fournies par cette méthode qu'on associe aux caractéristiques spécifiques en fatigue du matériau.

Raisonnons sur la dernière courbe d'évolution de $\Delta K_{\mathrm{II}}$ en fonction de la taille de fissure. Le rail est soumis à un chargement hertzien où $p_{0}=1,5 \mathrm{GPa}$ et $q_{0}=0,3 p_{0}$ avec une largeur de contact $2 c=5 \mathrm{~mm}$ et à des contraintes résiduelles de compression $\sigma_{\text {res }}=-300 \mathrm{MPa}$ : le coefficient de friction entre les faces de fissure vaut $\mu_{\mathrm{f}}=0,3$.

Cette courbe, tracée figure 15 et que nous noterons $\Delta K_{\mathrm{II}}=f(\mathrm{a})$, possède la forme générale évoquée à plusieurs reprises : montée rapide suivie d'un maximum $\Delta K_{\mathrm{II}}^{\max }$ (environ 10,2 $\mathrm{MPa} \sqrt{m}$ ) correspondant à une certaine taille de fissure (environ $2 \mathrm{~mm}$ ), puis décroissance. Cette décroissance résulte du fait que la fissure pénètre dans des couches du matériau de moins en moins soumises au cisaillement de contact.

Les caractéristiques de propagation du matériau sont le seuil de fissuration $\Delta K_{\text {IIS }}$ (on prendra ici $\Delta K_{\text {IIS }}=$ $6 \mathrm{MPa} \sqrt{m}$ pour illustrer le comportement) et les coefficients $C$ et $m$ (on prendra ici $C=5,02 \times 10^{-44}$ et $m=4,90$ respectivement) de la loi de Paris en cisaillement qui donne le taux de croissance par cycle de la fissure :

$$
\frac{\mathrm{d} a}{\mathrm{~d} N}=C \Delta K_{\mathrm{II}}^{m}
$$

On note $a_{0}$ la taille de fissure correspondant à $\Delta K_{\text {IIS }}$ (environ $0,6 \mathrm{~mm}$ ) et nous supposons que l'amorce de fatigue a une taille de départ $a_{\mathrm{i}}(0,82 \mathrm{~mm})$ supérieure à $a_{0}$, sans quoi l'amorce est un défaut court ne risquant pas de se propager selon le mécanisme envisagé de fissuration en mode II.

Dans ces conditions, la loi de Paris conduit par intégration à l'expression du nombre de cycles $N$ pour une taille de fissure a donnée :

$$
N(a)=\int_{a_{i}}^{a} \frac{\mathrm{d} a}{C f(a)^{m}}
$$

En inversant ce résultat, on obtient la loi d'avancée de fissure $a(N)$ en fonction du nombre de cycles $N$ comme le montre la figure 16 . 


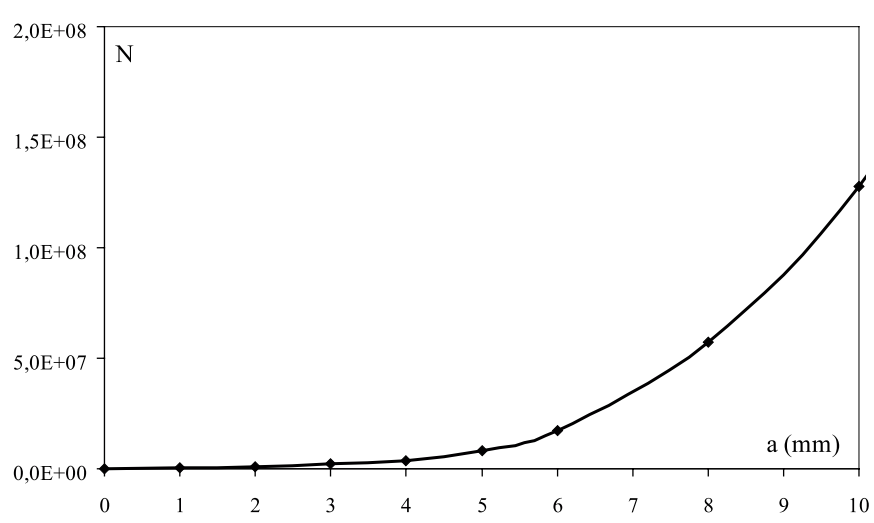

Fig. 16. Exemple de loi d'avancée de fissure.

L'issue de cette propagation repose sur une alternative entre deux situations extrêmes :

- soit la taille a parvient à une valeur pour laquelle l'embranchement en mode d'ouverture devient possible. La résolution de l'éventualité d'une bifurcation est liée non seulement à $K_{\text {II }}$ que nous savons calculer, mais également à $K_{\mathrm{I}}$ qui dépend d'un grand nombre de facteurs, dont notamment la pression de fluide à l'intérieur de la fissure, l'inclinaison de celle-ci, la rugosité des faces, la composante de flexion, etc. Pour une analyse plus complète de cet aspect, on peut se rapporter à la référence [1] ainsi qu'aux travaux de Bower [5] et de Dubourg et Villechaise [10].

- soit la fissure a pu atteindre une taille $a_{1}$ (environ $7 \mathrm{~mm}$ ) pour laquelle $\Delta K_{\text {II }}$ est redescendu au niveau de $\Delta K_{\text {IIS }}$, sans que la bifurcation se soit produite. Alors la fissure est bloquée dans sa progression puisque $\Delta K_{\text {II }}$ ne peut plus que décroître. C'est bien sûr cette situation qui est souhaitée, et nous avons vu qu'elle repose essentiellement sur la loi $\Delta K_{\mathrm{II}}=f(a)$ dont nous avons exposé la démarche d'analyse.

Pratiquement, la valeur de $\Delta K_{\mathrm{II}}^{\max }$ conditionne l'issue de la propagation en bifurcation ou en blocage. À partir du critère de bifurcation en contraintes normales maximales, voir Lemaitre [11], on sait que si $\Delta K_{\mathrm{II}}^{\max }<$ $\sqrt{2} \Delta K_{\mathrm{IS}}$, on ne risque pas la bifurcation en cisaillement. Dans notre cas d'étude, $\Delta K_{\mathrm{II}}^{\max }=10,2 \mathrm{MPa} \sqrt{m}$ et si le seuil $\Delta K_{\text {IS }}$ caractéristique du rail est de $8 \mathrm{MPa} \sqrt{m}$ (valeur courante), nous pouvons admettre que le blocage de la fissure est très probable.

Sur le plan du matériau, il est important de connaître avec précision les caractéristiques de la loi de Paris en cisaillement car une légère incertitude sur les données du matériau peut se traduire par une erreur importante sur les résultats de la modélisation effectuée.

La littérature comporte peu d'éléments à ce sujet. On peut toutefois citer les travaux de Doquet [12] qui a mis au point un processus expérimental avec suivi du déplacement latéral de faces de fissure sous MEB, ce qui a permis de caractériser un certain nombre de nuances d'acier et d'étudier leur comportement en mode II.
Nous pouvons rappeler à ce sujet que la méthode de calcul proposée permet de déterminer simplement le déplacement en chaque point d'une éprouvette, et que le calcul inverse qui consiste à déterminer la distribution des dislocations à partir des déplacements mesurés des points situés sur les faces de la fissure peut conduire directement au $\Delta K_{\text {II }}$ tout en donnant la répartition des coefficients de frottement entre faces. Cette méthode, judicieuse sur le plan de la modélisation, pourrait ainsi l'être tout autant sur le plan expérimental.

\section{Conclusion}

La phase de propagation en cisaillement qui succède à celle d'amorçage en fatigue de contact des rails, dans le cas du « head-check» notamment, est gouvernée par la variation $\Delta K_{\text {II }}$ du facteur d'intensité de contraintes en mode II dont dépend l'avancée de fissure.

La méthode des dislocations permet de modéliser en $2 \mathrm{D}$ cet endommagement dans chaque cas spécifique d'amorces initiales observées et de prévoir la durée en nombre de cycles pour atteindre une taille $a$ de fissure donnée, ou pour parvenir au blocage de la fissure. Cette dernière opportunité est offerte par la forme de la courbe $\Delta K_{\mathrm{II}}(a)$ qui présente systématiquement une phase décroissante qui peut permettre à $\Delta K_{\text {II }}$ de repasser endessous du niveau $\Delta K_{\text {IIS }}$. Par ailleurs, nous avons vu que la réalité $3 \mathrm{D}$ peut être correctement approchée par cette méthode, en déterminant des coefficients de passage obtenus par comparaison avec des cas 3D figurant dans des manuels spécialisés.

Parmi les facteurs augmentant $\Delta K_{\mathrm{II}}$, donc favorables à la propagation des fissures, on retient une orientation non verticale et le voisinage de fissures. Quant aux facteurs inhibiteurs, on retient essentiellement le frottement entre les faces de la fissure qui, s'il ne se manifestait pas, conduirait à une ruine rapide, ainsi qu'un état de contraintes résiduelles de compression agissant en synergie avec le frottement.

La connaissance précise des caractéristiques de propagation, seuil $\Delta K_{\text {IIS }}$ et paramètres $C$ et $m$ de la loi de Paris en mode II, est indispensable pour parvenir à une modélisation suffisamment précise. Là encore, la méthode des dislocations pourrait être mise à profit pour exploiter les expérimentations sur éprouvettes de cisaillement.

\section{Références}

[1] K.J. Miller, Structural integrity - Whose responsability? Ordinary meeting of The Institution of Mechanical Engineers, London, 12-9-2001

[2] A. Galtier, Y. Bourdon, Modèle de calcul des rails à la fatigue, Journées de Printemps de la SF2M, Senlis, mai 1998

[3] N.I. Muskhelishvili, Some basic problems of the mathematical theory of elasticity, Moscow, 1954, pp. 298-315, 91-413 
[4] Y. Murakami, Stress intensity factors handbook, Pergamon Press

[5] A.F. Bower, The influence of crack face friction and trapped fluid on surface initiated rolling contact fatigue cracks, Trans. ASME, J. Lubr. Technol. 110 (1988) 704-711

[6] F. Erdogan, G.D. Gupta, T.S. Cook, Method of analysis and solutions of crack problems, Noordhoff International Publishing, 1973, pp. 368-425

[7] D.A. Hills, M. Comninou, A normally loaded half-plane with an edge crack, Int. J. Solid Structures 21 (1985) $399-410$
[8] S. Krenk, On the use of the interpolation polynomial for solutions of integral equations, Quart. J. Appl. Math. 32 (1975) 479-484

[9] S.D. O'Regan, The driving force for mode II crack growth under rolling contact, Wear 101 (1985) 333-346

[10] M.C. Dubourg, B. Villechaise, Analysis of multiple fatigue cracks, J. Tribology (1991)

[11] J. Lemaitre, J.L. Chaboche, Mécanique des matériaux solides, Dunod, pp. 497-499

[12] V. Doquet, Étude de la propagation des fissures de fatigue en mode II avec prise en compte du frottement entre les flancs de la fissure, Mécanique Industrielle et Matériaux 50 (1997) 3697 